\title{
Trellis Coding of Quadrature Frequency/Phase Modulated Signals
}

\author{
Shalini S. Periyalwar, Member, IEEE, and Solomon Fleisher, Senior Member, IEEE
}

\begin{abstract}
In this paper, we explore the trellis coding of continuous phase QFPM (CPQFPM) signal sets and continuous phase FPM (CPFPM) signal sets, which are embodiments of the quadrature biorthogonal modulation (QBOM) technique. Conventional TCM and multiple TCM schemes with these modulation formats are examined using both the AWGN channel and the Rician fading channel design. Asymptotic coding gains in $d^{2}($ free $)$ are tabulated for trellis-coded rate $\frac{3}{4}, \frac{5}{6}$, and $\frac{6}{7}$ QFPM schemes, in comparison with uncoded modulations (8AMPM, 32AMPM) and other trellis-coded modulations [TCM (2FSK/ 4PSK, 16QAM, 64QAM), MTCM (2FSK/8PSK)] of equivalent throughput rate. Performance gains on the Rician fading channel are demonstrated by increased values of the design parameters for this channel, namely symbol diversity $L_{\min }$ and branch distance product $P$.
\end{abstract}

\section{INTRODUCTION}

$\mathrm{T}$ RELLIS coded modulation (TCM) [1], a technique of channel coding proposed by Ungerboeck [2], has been extensively investigated for a number of applications and with a variety of modulation formats. TCM techniques with MPSK signal sets have demonstrated coding gains on the AWGN [2] channel and the Rician fading channel [3]. Coded frequency/phase modulation (FPM) formats have been shown to provide performance gains on the AWGN channel [4] and the Rician fading channel [5]. Multidimensional trellis codes with lattice-type (QAM) signal constellations have been analyzed for performance on the AWGN channel by Ungerboeck [6], Forney [7], Wei [8], and others. Multidimensional trellis codes in the form of multiple TCM (MTCM) have been proposed by Divsalar and Simon for AWGN channels [9] and for the Rician fading channel [10], [11]. Trellis-coded multidimensional MPSK schemes for the AWGN channel have also been studied in [12].

A general schematic diagram of a TCM encoder-modulator is shown in Fig. 1. The redundancy in signal space is introduced by encoding $\tilde{m}$ of the $m$ input bits by using a rate $\tilde{m} /(\tilde{m}+1)$ convolutional encoder, and then mapping the $(\tilde{m}+1)$ bits at the convolutional encoder output to select a subset of a redundant $2^{m+1}$-ary signal set. The remaining $m-\tilde{m}$ bits are used to select one of the $2^{m-\tilde{m}}$ signals in the subset for transmission. The mapping of bit

Manuscript received September 2, 1991; revised June 30, 1992

The authors are with the Department of Electrical Engineering. Technical University of Nova Scotia, Halifax NS B3J 2 X4 Canada.

IEEE Log Number 9203040 sequences (termed mapping by set partitioning) into signals from an $M$-ary alphabet (where $M=2^{m+1}$ ) is unique to combined coding and modulation schemes such as TCM, and is performed to meet different design criteria depending on the type of channel that is involved. The signal set employed in the TCM scheme may be two-dimensional (e.g., MPSK, QAM) or multidimensional [e.g., 4-D QAM, MFSK, frequency and phase modulation (FPM)].

Multidimensional modulations may be realized by the transmission of a series of constituent $1-D$ or $2-D$ symbols. Thus, for example, 4- $D$ and 8- $D$ modulations are obtained from 2-D modulations by the transmission of groups of 2 or 4 symbols per trellis transition. By applying this technique to trellis coding of $M$-ary PSK and $M$-ary AM signal sets, Divsalar and Simon introduced the concept of multiple trellis coded modulation (MTCM) [9]. In the MTCM encoder-modulator [9], the output bits from the rate $m / m+1$ encoder in Fig. 1 are assigned signal $k$-tuples $(k>1)$ from an expanded signal set. The essential difference between TCM and MTCM is in the multiplicity $k$ of signals available in each transmission interval at the output of the encoder-modulator. For the conventional TCM scheme, $k$ is equal to 1 .

The design criterion for trellis codes on the AWGN channel is well known to be the maximization of $d^{2}$ ( free). On the Rayleigh fading channel (with no direct signal component), under the conditions of ideal interleaving/ deinterleaving, the criteria for optimum code design have been defined as the length $L_{\min }$ (defined as the number of symbols at nonzero Euclidean distance) of the shortest error event path, and the product of branch distances $P$ along that path [10]. On the Rician fading channel with direct signal components, the performance is affected by all three quantities mentioned above $\left[d^{2}\right.$ (free), $L_{\min }$, and $\left.P\right]$.

As an example of TCM and MTCM schemes, Fig. 2 illustrates the signal assignments to a two-state trellis (designed for the AWGN channel) for a rate of $\frac{1}{2}(k=1)$ TCM scheme and a rate $\frac{2}{3}(k=2)$ MTCM scheme, both using QPSK signals. The trellis structure consists of $2^{m}$ transitions from each state in the trellis diagram, with each transition being represented by $k$ symbols, chosen in such a way as to meet the design criteria. To represent all possible combinations of the $m+1$ bits at the encoder output $2^{m+1}$ distinct $k$-tuples are required. Each of the trellis transitions in the rate $\frac{1}{2}$ scheme is assigned one QPSK signal while each of the trellis transitions in the rate $\frac{2}{3}$ scheme 


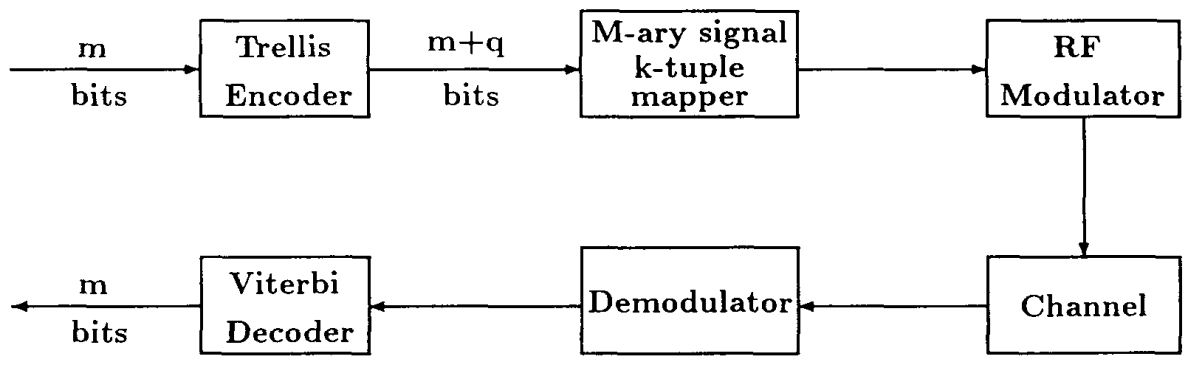

Fig. 1. Encoder-modulator for TCM.

0
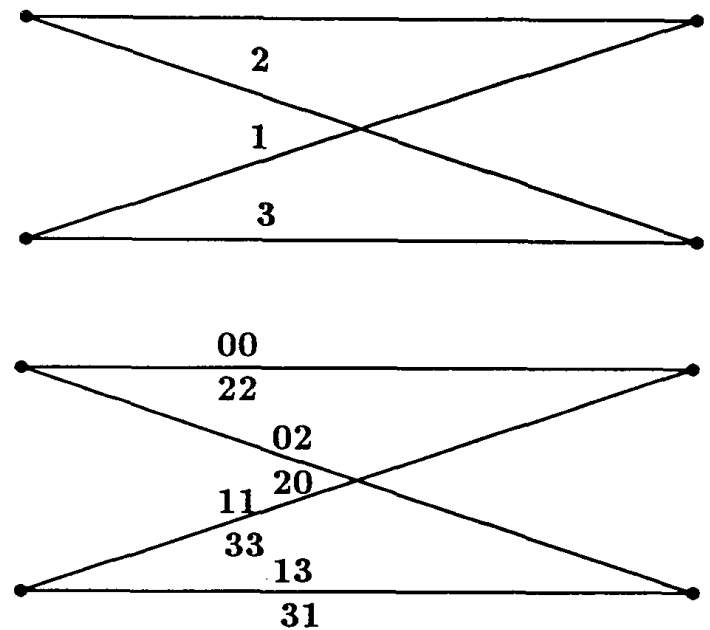

Fig. 2. Two-state trellis for a rate ${ }_{2}^{1}$ TCM scheme and a rate $\frac{2}{3}$ MTCM scheme.

is assigned two QPSK signals. The MTCM scheme offers the advantages of increased $d^{2}$ (free) when compared to the TCM scheme, yielding a performance gain on the AWGN channel. It also has a larger time diversity $(k=$ 2) with respect to the former $(k=1)$, and thus attains improved performance on the Rayleigh or Rician fading channel $\left(L_{\min }=k\right)$. Note, however, that the four-dimensional MTCM scheme has more transitions per trellis state than the two-dimensional TCM scheme, which contributes to an increase in error coefficient. The throughput rate of both schemes is $m / k=1 \mathrm{~b} / \mathrm{s} / \mathrm{Hz}$.

While the time orthogonality (multiplicity) in MTCM schemes results in increased dimensionality $(=2 k$, if twodimensional modulation is employed in each time interval) of the trellis-coded schemes, multidimensional modulations realized by frequency orthogonality (i.e., the use of $N(\geq 2)$ orthogonal frequencies such as in FPM) have been used with TCM [4] and MTCM [5] schemes to yield performance gains on the AWGN channel and the Rician fading channel. In these schemes, the increased dimensionality is due to both $N$ and $k$, and equals $2 N k$. MTCM schemes with FPM signal sets, thus, employ a combination of time and frequency orthogonality to increase dimensionality. One may argue that the increased dimen- sionality resulting from frequency orthogonality may be accompanied by a price in bandwidth and, consequently, a reduced throughput rate. For example, compare the rate $\frac{4}{5}(k=2)$ MTCM schemes employing 8PSK signals $(N=$ l) and 2FSK/4PSK FPM signals $(N=2)$. By defining the throughput rate as $r=m / N k,{ }^{1}$ it may be argued that the throughput rates of these schemes is $2 \mathrm{~b} / \mathrm{s} / \mathrm{Hz}$ and $\mathrm{l} \mathrm{b} / \mathrm{s} / \mathrm{Hz}$, respectively, and therefore any gain in $d^{2}$ (free) obtained by the MTCM/FPM scheme with respect to the MTCM/8PSK scheme is not without penalty. However, as observed in [4], the bandwidth occupancy of the FPM schemes in terms of $99 \%$ (or a number of other percentages [14]) signal power containment is the same or lower than that of MPSK schemes and, under this definition of bandwidth, the comparison of the two schemes is valid without penalty in the throughput rate. A similar analysis will be presented for the QFPM and CPQFPM schemes to be discussed.

In this paper, the technique of quadrature biorthogonal modulation (QBOM) [15] in combination with trellis coding is examined for performance on the AWGN channel and the Rician fading channel. Quadrature frequency/ phase modulated (QFPM) [16] and its continuous phase modification (CPQFPM) [15], which are embodiments of the QBOM technique, are considered. The constant envelope versions of QFPM and CPQFPM, the CEQFPM and CPFPM schemes [17], respectively, are of particular interest for satellite communication channels. It turns out that the CEQFPM scheme is, in fact, a $4 N$-ary $N F S K /$ 4PSK scheme, and CPFPM consequently can be viewed as a continuous-phase version of $N F S K / 4 P S K$. The $(2 N)^{2}-$ ary QFPM signal set is derived as a quadrature-carrier sum of two biorthogonal $N$-dimensional $N F S K / 2 P S K$ signal sets. In Section II of this paper, the QFPM technique is discussed. Section III covers the performance analysis of conventional TCM schemes and MTCM schemes employing QFPM-type signals. Trellis-code designs for the AWGN channel and the Rayleigh fading channel are considered, and the design parameters $d^{2}$ (free), $L_{\text {min }}$, and $P$ are evaluated for all codes. Asymptotic coding gains are demonstrated over uncoded modulation and other trelliscoded modulations of equivalent throughput rate. Section IV contains the discussion.

'This approximation is based on the Nyquist bandwidth and is crude, as far as $N$ is concerned [13]. 


\section{Quadrature Frequency/Phase Modulation FORMATS}

Biorthogonal modulation [18] and quadrature-carrier multiplexing are two well-known techniques in digital communications. Both these techniques have been incorporated into a novel modulation format [15], referred to as quadrature biorthogonal modulation (QBOM).

A $2 N$-ary biorthogonal signal set [18] comprises two polar signals on each of $N$ orthogonal carriers. In particular, a biorthogonal signal set can be generated by combined frequency/phase modulation of the type NFSK/ 2PSK, with the orthogonal signals $\cos \omega_{i}(t), i=1, \cdots$, $N$ defining the orthogonal vectors of the $N$-dimensional signal space.

By introducing the concept of quadrature-carrier multiplexing into this technique, a $2 \mathrm{~N}$-dimensional signal space may be realized by utilizing, along with the cosinusoidal vectors defined above, their sinusoidal counterparts. Quadrature-carrier multiplexing of two biorthogonal signal sets yields a new modulation technique named quadrature frequency/phase modulation, acronymed QFPM. The frequencies in each of the two biorthogonal signal sets are offset from the center frequency $\omega_{c}$ by an integer multiple of $\omega_{d}=\pi t / T$ (frequency modulation in$\operatorname{dex} h=1)$.

The $(2 N)^{2}$-ary QFPM signal set occupies the vertices of a $2 \mathrm{~N}$-dimensional hypercube, and can be expressed as

$$
s(t)= \pm \cos \omega_{i} t \pm \sin \omega_{j} t \quad i, j=1,2, \cdots, N .
$$

The QFPM signal set is a constant energy signal set, but has a nonconstant envelope in the general case of $i \neq j$. When the same frequency $\omega_{i}=\omega_{j}$ is used for both the cosinusoidal and sinusoidal components in any signaling interval, the QFPM signal set reduces to a $4 N$-ary constant envelope QFPM (CEQFPM) signal set. It can be readily seen that the CEQFPM signal set is, in fact, a frequency and phase modulated (FPM) signal set of the type NFSK/4PSK. ${ }^{2}$

The CPQFPM and CPFPM techniques described in [15], [17] are continuous-phase modifications of QFPM and CEQFPM, respectively, providing rapid rolloff of sidelobes in the power spectrum at the expense of a double-frequency modulation index $(h=2)$.

For the unit-energy QFPM $(N=2)$ or the CEQFPM $(N=2)$ signal sets, the signal space is four-dimensional, and the orthonormal basis functions are defined in (3)

\footnotetext{
'The $N F S K / 4 P S K$ signal set belongs to the class of $M$-ary $N$ FSK//PSK FPM signals $(l=M / N=4,8$. etc.) which have been investigated with TCM [4] and MTCM [5]. The general definition for the FPM signal set is given as

$$
s(t)=\sqrt{\frac{2}{T}} \cos \left[\left(\omega_{c} \pm b h \pi / T\right) t-\phi_{l}\right]
$$

where $T$ is the symbol duration, $b=1,3, \cdots,(N-1), \phi_{i} \in(0,2 \pi / l$, $\cdots, 2(l-1) \pi / l)$, and $h$ takes on any suitable value (e.g., $h=0.5,1)$. The signal space is $2 N$-dimensional, and is defined in [4] for the fourdimensional 2 FSK/lPSK schemes. For the class of $N F S K / 4 P S K$ signals discussed in this paper, $h=1$.
}

$$
\begin{aligned}
& \psi_{1}=\sqrt{\frac{2}{T}} \cos \omega_{1} t ; \quad \psi_{2}=\sqrt{\frac{2}{T}} \sin \omega_{1} t ; \\
& \psi_{3}=\sqrt{\frac{2}{T}} \cos \omega_{2} t ; \quad \psi_{4}=\sqrt{\frac{2}{T}} \sin \omega_{2} t .
\end{aligned}
$$

The QFPM $(N=2)$ signal set contains the following signals:

$$
\begin{array}{rrr}
0: \cos \omega_{1} t+\sin \omega_{1} t & 1: \cos \omega_{1} t+\sin \omega_{2} t \\
4:-\cos \omega_{1} t+\sin \omega_{1} t & 5:-\cos \omega_{1} t+\sin \omega_{2} t \\
8:-\cos \omega_{1} t-\sin \omega_{1} t & 9:-\cos \omega_{1} t-\sin \omega_{2} t \\
12: \cos \omega_{1} t-\sin \omega_{1} t & 13: \cos \omega_{1} t-\sin \omega_{2} t \\
2: \cos \omega_{2} t+\sin \omega_{2} t & 3: \cos \omega_{2} t+\sin \omega_{1} t \\
6:-\cos \omega_{2} t+\sin \omega_{2} t & 7:-\cos \omega_{2} t+\sin \omega_{1} t \\
10:-\cos \omega_{2} t-\sin \omega_{2} t & 11:-\cos \omega_{2} t-\sin \omega_{1} t \\
14: \cos \omega_{2} t-\sin \omega_{2} t & 15: \cos \omega_{2} t-\sin \omega_{1} t
\end{array}
$$

The squared Euclidean distance between the signal point 0 and all other signal points is as follows:

$$
\begin{array}{rll}
d^{2}(0,0)=0 & d^{2}(0,2)=2 & d^{2}(0,1)=1 \\
d^{2}(0,3)=1 & d^{2}(0,4)=2 & d^{2}(0,6)=2 \\
d^{2}(0,5)=3 & d^{2}(0,7)=3 & d^{2}(0,8)=4 \\
d^{2}(0,10)=2 & d^{2}(0,9)=3 & d^{2}(0,11)=3 \\
d^{2}(0,12)=2 & d^{2}(0,14)=2 & d^{2}(0,13)=1 \\
d^{2}(0,15)=1 . & &
\end{array}
$$

This signal set occupies the vertices of a four-dimensional hypercube. The minimum squared Euclidean distance of this signal set is $d_{\min }^{2}=1$. The even-numbered signals of this 2QFPM signal set, corresponding to $\omega_{i}=\omega_{j}$, constitute the CEQFPM $(N=2)$ signal set with $d_{\min }^{2}=2$. The minimum squared Euclidean distance of the 16-ary QFPM $(N=2)$ signal set is higher than that for the 16-ary 2FSK/ 8PSK FPM signal set [4], [5], which has $d_{\text {min }}^{2}=0.5858$.

The QFPM $(N=4)$ signal set contains 64 signals. The constant envelope subset of the signals in this set is the 16-ary CEQFPM $(N=4)$ signal set. The value of $d_{\min }^{2}$ for the QFPM $(N=4)$ signal set remains the same as that for the QFPM $(N=2)$ signal set. The bandwidth requirement and the data rate are larger than for QFPM $(N=2)$.

The QFPM technique is discussed in detail in [14], wherein the topics of modulation/demodulation techniques, spectra, power and bandwidth efficiencies, and comparison with QAM/MPSK are addressed.

\section{Trellis Coding of QFPM Signals}

Set partitioning to meet the design criterion of $d^{2}$ ( free) for TCM schemes on the AWGN channel begins with the division of the signal set into subsets of odd-numbered and even-numbered signals. The signal points from each subset are assigned to trellis transitions from alternate states. 
In the MTCM design for the AWGN channel, each trellis transition is assigned multiple $(k>1)$ signals, which are obtained as Cartesian products of signals within a given subset [9]. Again, subsets of odd-numbered and even-numbered signal $k$-tuples are assigned to alternate trellis states. On the Rayleigh fading channel, to meet the design criteria of length $L_{\min }$ and the branch distance product $P$ for MTCM schemes, the set partitioning is carried out to ensure that parallel transitions in particular are assigned multiple signals which differ in all $k$ positions (to provide $L_{\min }=k$ ), in such a way that the branch distance product is maximized as well [11]. In this paper, we have applied the two set partitioning techniques to MTCM schemes with $k=2$.

Here, TCM and MTCM $(k=2)$ schemes are investigated for performance with QFPM signal sets. For all codes, including the TCM and MTCM codes designed for the AWGN channel $d^{2}$ (free), $L_{\text {min }}$, and $P$ are tabulated in Tables II and V. The latter two computations are added to trellis codes designed for the AWGN channel to highlight the merits of the fading channel design where applicable or, in some cases, to illustrate the merits of the TCM schemes over their MTCM counterparts. From the results tabulated in Table III for MTCM schemes, we arrive at the conclusion that, for QFPM signal sets, set partitioning for the Rayleigh fading channel is also optimum for the AWGN channel. A similar conclusion was reached in the analysis of multiple trellis-coded FPM signals in [5].

Wherever possible, asymptotic coding gains are computed with respect to uncoded and coded signal sets with nonconstant envelope (QAM, AM-PM) for coded QFPM schemes, and with constant envelope (MPSK, FPM) for coded CEQFPM schemes. In some cases, asymptotic coding gains are also reported with respect to constant envelope signal sets.

In the comparison between coded QFPM and other modulation formats, it is always assumed that the bandwidth occupancy and, consequently, the throughput rate of the relevant signal sets are the same. With respect to FPM signal sets, this is true when the number of frequencies $N$ and the frequency modulation index $h$ are the same in QFPM and FPM. It can be shown [14] that, under certain conditions, this assumption is also valid with respect to QAM and MPSK signal sets. For a given bit rate, the bandwidth of QFPM is perceived to be wider than that of QAM (or MPSK). However, the normalized $B T_{b}$ that captures $99 \%$ of the total power, $B_{99}$, is approximately the same for QFPM with $N=2,4$, and 8, and QAM (or MPSK). Clearly, if the transmission bandwidth is limited to a value $B_{x}<B_{99}$, the throughput rate of QFPM will be less than that of QAM; similarly, if $B_{x}>B_{99}$, the throughput rate of QFPM will be higher than that of QAM. The same analysis holds for the comparison between CEQFPM and MPSK.

The situation is more favorable for CPQFPM. The main lobe of the power spectral density of CPQFPM is wider than that of QFPM for any given $N$, but the sidelobes fall off more rapidly. As a result, the bandwidth efficiency of
TABLE I

Fractional Power Containment and Bandwidths Corresponding to EQUAL BANDWIDTH EFFICIENCY FOR CPQFPM AND QAM (MPSK) WITH A Signal SET Size $M=4 N^{2}$, AND For CPFPM AND QAM (MPSK) WITH $M=4 N$

\begin{tabular}{cccc}
\hline & & \multicolumn{2}{c}{ Bandwidth $B$} \\
\cline { 3 - 4 }$N$ & $S$ & $M=4 N^{2}$ & $M=4 N$ \\
\hline 2 & $91 \%$ & $0.575 r_{b}$ & $1.18 r_{b}$ \\
4 & $95 \%$ & $0.72 r_{b}$ & $1.87 r_{b}$ \\
8 & $97.5 \%$ & $1.04 r_{b}$ & $3.16 r_{b}$ \\
\hline
\end{tabular}

TABLE II

$d^{2}(f r e e), L_{\mathrm{min}}$, AND $P$ FOR THE TCM SCHEMES

\begin{tabular}{|c|c|c|c|c|}
\hline Description & States & $d^{2}($ free $)$ & $L_{\min }$ & $P$ \\
\hline Rate: $\frac{2}{3}$ & 2 & 4 & 1 & 4 \\
\hline \multirow[t]{3}{*}{ Signal Set: CPFPM $(N=2)$} & 4 & 4 & 2 & 4 \\
\hline & 8 & 6 & 3 & 16 \\
\hline & 16 & 6 & 3 & 16 \\
\hline Rate: ${ }_{4}^{3}$ & 2 & 2 & 1 & 2 \\
\hline \multirow[t]{3}{*}{ Signal Set: CPQFPM $(N=2)$} & 4 & 3 & 1 & 4 \\
\hline & $8_{\mathrm{HC}}$ & 4 & 1 & 4 \\
\hline & 16 & 4 & 2 & 4 \\
\hline Rate: $\frac{3}{4}$ & 2 & 2 & 1 & 2 \\
\hline \multirow[t]{3}{*}{ Signal Set: CPFPM $(N=4)$} & 4 & 4 & 1 & 4 \\
\hline & $8_{\mathrm{HC}}$ & 6 & 1 & 4 \\
\hline & 16 & 6 & 2 & 4 \\
\hline Rate: ${ }_{6}^{5}$ & 2 & 2 & 1 & 2 \\
\hline \multirow[t]{3}{*}{ Signal Set: CPQFPM $(N=4)$} & 4 & 2 & 1 & 2 \\
\hline & 8 & 2 & 1 & 2 \\
\hline & 16 & 2 & 1 & 4 \\
\hline
\end{tabular}

CPQFPM exceeds that of QAM (or MPSK) even in terms of bandwidths that correspond to a fraction of the total power considerably smaller than $99 \%$. Table I contains, for various values of $N$, the fraction of total power corresponding to equal bandwidth efficiencies of CPQFPM (and CPFPM) on the one hand, and QAM (or MPSK) on the other. Clearly, with a bandwidth corresponding to a larger fractional power containment than that indicated in Table I, the bandwidth efficiency of CPQFPM and CPFPM is higher than that of QAM (or MPSK). The bandwidths in terms of $r_{b}$, corresponding to the indicated fractions of total power, are also given in Table I. The bandwidths are calculated for signal sets of size $M=4 N^{2}$ corresponding to CPQFPM and $M=4 N$ corresponding to CPFPM, as well as QAM and MPSK signal sets of the same size $M$.

Thus, while the performance of coded QFPM and CEQFPM as well as their continuous phase versions (CPQFPM and CPFPM) is the same in terms of $d^{2}$ (free), $L_{\min }$, and $P$, the continuous phase modulation schemes make realizable the assumption of equal (or larger) throughput for a larger range of bandwidth occupancy. For this reason, the following analysis refers to CPQFPM and CPFPM schemes rather than QFPM and CEQFPM. The results of the analysis hold for QFPM and CEQFPM 
TABLE III

Coding Gains in $d^{2}$ (free) for the Trellis-Coded Rate ${ }_{4}^{3}$ QFPM Scheme

\begin{tabular}{lcccc}
\hline \multicolumn{1}{c}{ Description } & States & $\begin{array}{c}\text { Gain }(\mathrm{dB}) \\
\text { Versus } \\
\text { Uncoded } \\
\text { 8AMPM }\end{array}$ & $\begin{array}{c}\text { Gain }(\mathrm{dB}) \\
\text { Versus } \\
\text { TCM } \\
\text { 2FSK/8PSK }\end{array}$ & $\begin{array}{c}\text { Gain }(\mathrm{dB}) \\
\text { Versus } \\
\text { TCM } \\
\text { 16QAM }\end{array}$ \\
\hline Rate: ${ }^{3}$ & 2 & 3.98 & 0.0 & 2.3 \\
Signal Set: CPQFPM $(N=2)$ & 4 & 5.74 & 1.76 & 2.7 \\
& 8 & 6.99 & 1.9 & 3.01 \\
& 16 & 6.99 & 1.9 & 2.25 \\
\hline
\end{tabular}

when the utilized transmission bandwidth captures at least $99 \%$ of the total power; otherwise, some loss in bandwidth efficiency should be taken into account.

\section{A. TCM Schemes}

Table II contains the values of $d^{2}$ (free), $L_{\min }$, and $P$ for the following TCM schemes.

1) Rate $\frac{2}{3}$ code: This code requires an expanded 8 -ary signal set. The constituent constant envelope CPFPM $(N$ $=2$ ) signal subset of the CPQFPM $(N=2)$ signal set may be used. For this code, the values of $d^{2}$ (free) are reported by Padovani and Wolf [4] and are reproduced in Table II for reference. Table II also contains the values of $L_{\min }$ and $P$. This code is compared with the uncoded QPSK scheme and the rate $\frac{2}{3}$ trellis-coded 8PSK scheme in [4].

2) Rate $\frac{3}{4}$ code: For this code, an expanded 16-ary signal set is used. The signal set is partitioned as follows:

$$
\begin{array}{ll}
A=(0,4,8,12) ; & B=(2,6,10,14) ; \\
C=(1,5,9,13) ; & D=(3,7,11,15) .
\end{array}
$$

Two 16-ary signal sets are considered: The CPQFPM $(N=2)$ signal set, and the CPFPM $(N=4)$ signal set. The signal space structure of the QFPM $(N=2)$ signal set is given in Section II. For the two-state trellis shown in Fig. 3, the parallel transitions determine $d^{2}$ (free) and $d^{2}($ free $)=2, L_{\min }=1$, and $P=2$. The two-branch error event has $d^{2}=3$.

For the four-state trellis shown in Fig. 4, subsets assigned are as follows:

$$
\begin{aligned}
& A_{0}=(0,8) ; \quad A_{1}=(4,12) ; \quad B_{0}=(2,10) ; \\
& B_{1}=(6,14) ; \quad C_{0}=(1,9) ; \quad C=(5,13) ; \\
& D_{0}=(3,11) ; \quad D_{1}=(7,15) \text {. }
\end{aligned}
$$

The squared free distance is determined by the twobranch error event with $d^{2}=3$. The distance between the parallel transitions is $4 . L_{\min }$ and $P$ are determined by the parallel transitions.

For the eight-state half-connected trellis (since the free distance is not determined by the parallel transitions in the four-state trellis), $d^{2}$ (free) $=4$ due to the parallel transitions and due to the two-branch error event, $L_{\mathrm{min}}$, and $P$ remain the same as for the four-state code. For the sixteen-state trellis, $d^{2}$ (free) $=4$ due to the two-branch er-

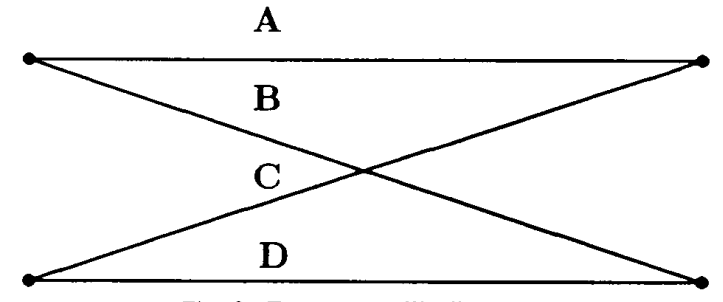

Fig. 3. Two-state trellis diagram

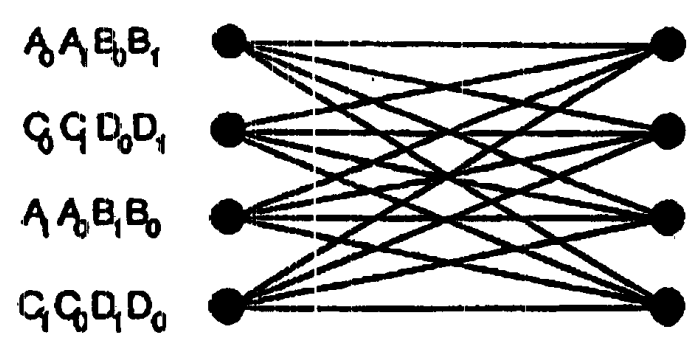

Fig. 4. Four-state trellis diagram

ror event, $L_{\min }=2$, and $P=4$. To achieve a larger value of $d^{2}$ ( free), the number of states has to be increased until the two-branch error event disappears. This is possible when 32 or more states are used.

The values of $d^{2}$ ( free), $L_{\min }$, and $P$ are listed in Table II for this code. Comparison with uncoded 8AMPM, trellis-coded 2FSK/8PSK [4], and trellis-coded 16QAM is given in Table III. With respect to uncoded 8AMPM, coding gains up to $6.99 \mathrm{~dB}$ are observed for the eight-state trellis. With respect to trellis-coded 2 FSK/8PSK, coding gains of $1.76 \mathrm{~dB}$ for the four-state trellis and $1.9 \mathrm{~dB}$ for the eight-state and sixteen-state trellises are observed. With respect to trellis-coded 16QAM [2], coding gains up to $3.01 \mathrm{~dB}$ for the eight-state trellis are observed.

Alternately, the constant envelope CPFPM $(N=4)$ signal subset of the CPQFPM $(N=4)$ signals may be employed for this code. The values of $d^{2}$ ( free) are higher when compared to the use of CPQFPM $(N=2)$ signals. However, the throughput is lower in this case. This is a typical case of tradeoff in throughput for power. Also, the constant envelope characteristic of CPFPM is of relevance to satellite communication channels.

3) Rate $\frac{5}{6}$ code: This code requires the 64-ary CPQFPM $(N=4)$ signal set. The two-state trellis has 16 parallel 
TABLE IV

Coding Gains in $d^{2}$ (free) for the Trellis-Coded Rate ${ }_{6}^{5}$ QFPM Scheme

\begin{tabular}{lccc}
\multicolumn{1}{c}{ Description } & States & $\begin{array}{c}\text { Gain }(\mathrm{dB}) \\
\text { Versus } \\
\text { Uncoded 32AM-PM }\end{array}$ & $\begin{array}{c}\text { Gain (dB) } \\
\text { Versus } \\
\text { TCM/64QAM }\end{array}$ \\
\hline Rate: 5 & 2 & 10.2 & - \\
Signal Set: CPQFPM $(N=4)$ & 4 & 10.2 & 7.41 \\
& 8 & 10.2 & 6.41 \\
& 16 & 10.2 & 5.61 \\
\hline
\end{tabular}

transitions with a minimum distance of 2 . The distance between any two of the four subsets assigned to the twostate trellis is 1 . Therefore, both the parallel transitions and the two-branch error event have a minimum distance of 2. For the four-state and eight-state trellises, there are 8 and 4 parallel transitions, respectively, both with a minimum distance of 2 as well. For the sixteen-state trellis, the distance between the parallel transitions increases to 4 , but $d^{2}$ ( free) remains at 2 due to the two-branch error event. The number of states has to be increased to 128 or more to avoid this error event. In all of the above cases, $L_{\min }$ and $P$ given in Table II are due to the parallel transitions. In Table IV, this code is compared with uncoded 32AM-PM and coded 64QAM [2], yielding coding gains of 10.2 and $7.41 \mathrm{~dB}$ (four-state trellis) respectively.

\section{B. MTCM Schemes}

Table V contains the values of $d^{2}$ ( free), $L_{\min }$, and $P$ of the following MTCM schemes.

1) Rate $\frac{4}{5}$ code $(k=2)$ : This code requires 32 signal pairs, which may be obtained from a Cartesian product of CPFPM $(N=2)$ signal sets. This code has been discussed in [5] for 2FSK/4PSK signal sets. The results are reproduced in Table III of the AWGN and fading channel designs, for trellises up to sixteen states, for comparison with other codes discussed in this paper. The values of $d^{2}$ ( free) obtained with the AWGN channel design are the same as that for the rate $\frac{2}{3}$ TCM code discussed earlier. Multiple trellis coding offers advantages when the Rayleigh fading channel design is used, yielding a higher value of $d^{2}$ ( free ) for the sixteen-state trellis and a higher value of $L_{\min }$ for the two-state trellis. Clearly, for the eight-state and sixteen-state trellises, the rate $\frac{2}{3}$ TCM code has higher values of $L_{\min }$ due to the absence of parallel transitions, while the parallel transitions still prevailing in the rate $\frac{4}{5}$ MTCM code limit $L_{\min }$ to 2 .

2) Rate $\frac{5}{6}$ code $(k=2)$

AWGN channel design: This code has 32 transitions from each state, and a total of 64 unique signal pairs which may be obtained by a Cartesian product of CPFPM $(N=$ $2)$ signals are required. The signal set is first partitioned into sets $a=(0,2,4,6)$ and $b=(1,3,5,7)$. The Cartesian set products $A=a \times a, B=b \times b, C=a \times b, D$ $=B \times A$ defines the 64 unique signal pairs. For all these sets, the intraset distance is 2 . The interset distances are $d^{2}(A, B)=2$ and $d^{2}(A, C)=d^{2}(A, D)=4$.

For the two-state trellis with sets $A$ and $B$ assigned to
TABLE V $d^{2}($ free $), L_{\min }$, AND $P$ FOR THE MTCM $(k=2)$ SCHEMES

\begin{tabular}{|c|c|c|c|c|}
\hline Description & States & $d^{2}($ free $)$ & $L_{\min }$ & $P$ \\
\hline $\begin{array}{l}\text { Rate: }{ }_{5}^{4} \\
\text { Signal Set: CPFPM }(N=2) \\
\text { Design: AWGN }\end{array}$ & $\begin{array}{r}2 \\
4 \\
8 \\
16\end{array}$ & $\begin{array}{l}4 \\
4 \\
6 \\
6\end{array}$ & $\begin{array}{l}1 \\
2 \\
2 \\
2\end{array}$ & $\begin{array}{r}4 \\
4 \\
16 \\
16\end{array}$ \\
\hline $\begin{array}{l}\text { Rate: }{ }^{4} \\
\text { Signal Set: CPFPM }(N=2) \\
\text { Design: Fading } \\
\text { Uncoded: QPSK }\end{array}$ & $\begin{array}{r}2 \\
4 \\
8 \\
16\end{array}$ & $\begin{array}{l}4 \\
4 \\
6 \\
8\end{array}$ & $\begin{array}{l}2 \\
2 \\
2 \\
2\end{array}$ & $\begin{array}{r}4 \\
4 \\
16 \\
16\end{array}$ \\
\hline $\begin{array}{l}\text { Rate: }{ }^{5} \\
\text { Signal Set: CPFPM }(N=2) \\
\text { Design: AWGN }\end{array}$ & $\begin{array}{r}2 \\
4 \\
8 \\
16\end{array}$ & $\begin{array}{l}2 \\
4 \\
4 \\
4\end{array}$ & $\begin{array}{l}1 \\
1 \\
2 \\
2\end{array}$ & $\begin{array}{r}2 \\
4 \\
4 \\
16\end{array}$ \\
\hline $\begin{array}{l}\text { Rate: }{ }_{6}^{5} \\
\text { Signal Set: CPQFPM }(N=2) \\
\text { Design: Fading }\end{array}$ & $\begin{array}{r}2 \\
4 \\
8 \\
16\end{array}$ & $\begin{array}{l}4 \\
4 \\
4 \\
6\end{array}$ & $\begin{array}{l}2 \\
2 \\
2 \\
2\end{array}$ & $\begin{array}{r}3 \\
4 \\
4 \\
16\end{array}$ \\
\hline $\begin{array}{l}\text { Rate: } \stackrel{6}{7} \\
\text { Signal Set: CPQFPM }(N=2) \\
\text { Design: AWGN }\end{array}$ & $\begin{array}{r}2 \\
4 \\
8 \\
16\end{array}$ & $\begin{array}{l}2 \\
4 \\
4 \\
4\end{array}$ & $\begin{array}{l}1 \\
1 \\
2 \\
2\end{array}$ & $\begin{array}{l}2 \\
4 \\
4 \\
4\end{array}$ \\
\hline $\begin{array}{l}\text { Rate: } \frac{7}{8} \\
\text { Signal Set: CPQFPM }(N=2) \\
\text { Design: AWGN }\end{array}$ & $\begin{array}{r}2 \\
4 \\
8 \\
16\end{array}$ & $\begin{array}{l}2 \\
2 \\
3 \\
3\end{array}$ & $\begin{array}{l}1 \\
1 \\
2 \\
2\end{array}$ & $\begin{array}{l}2 \\
2 \\
4 \\
4\end{array}$ \\
\hline $\begin{array}{l}\text { Rate: } \frac{7}{8} \\
\text { Signal Set: CPQFPM }(N=4) \\
\text { Design: Fading }\end{array}$ & $\begin{array}{r}2 \\
4 \\
8 \\
16\end{array}$ & $\begin{array}{l}4 \\
4 \\
4 \\
6\end{array}$ & $\begin{array}{l}2 \\
2 \\
2 \\
2\end{array}$ & $\begin{array}{l}3 \\
3 \\
4 \\
4\end{array}$ \\
\hline
\end{tabular}

state 0 and sets $C$ and $D$ assigned to state $1, d^{2}$ ( free $)=$ 2 is determined by the parallel transitions. For the fourstate trellis, both the one-branch error event (parallel transitions) and the two-branch error event account for $d^{2}($ free $)=4$. For the eight-state and sixteen-state trellises, $d^{2}$ (free) remains at 4 due to the two-branch error event.

Fading channel design: The two-state trellis for this code has 16 parallel transitions, and to maintain $L_{\min }=k$ $=2$ a 16-ary signal set such as CPQFPM $(N=2)$ is required. The Cartesian products of the even-numbered and odd-numbered signal points yields 128 signal pairs, of which only 64 are required. The set partitioning is now carried out to obtain $L_{\min }=k=2$ and to maximize the branch distance product $P$. The two-state trellis is shown in Fig. 5, along with the assigned signal pairs. 


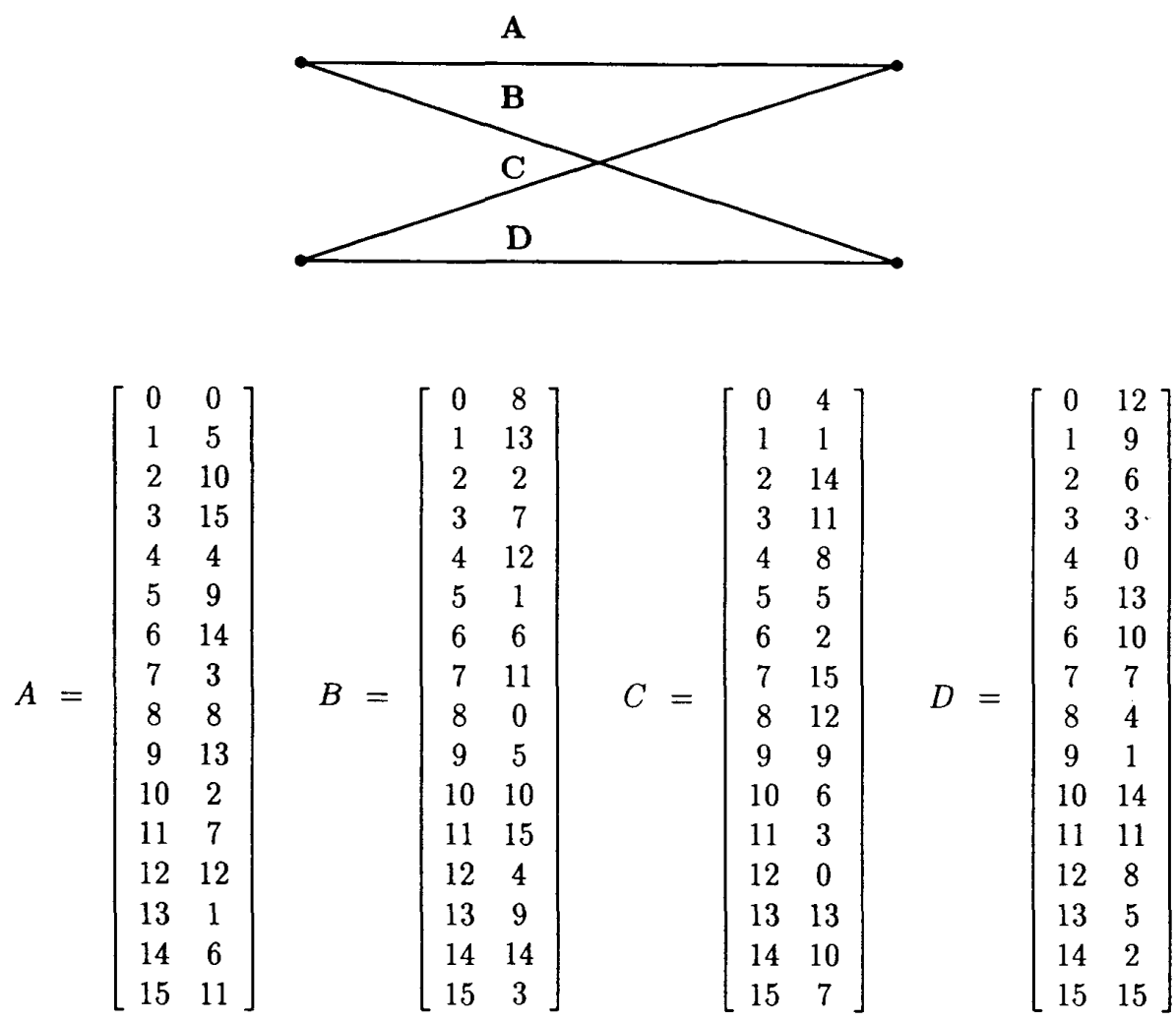

Fig. 5. Two-state trellis diagram for a rate $\frac{5}{6} \operatorname{MTCM}(k=2)$ scheme (fading design).

TABLE VI

Coding Gains in $d^{2}($ free $)$ For the Trellis-Coded Rate ${ }_{6}^{5}(k=2)$ QFPM SCHEME

\begin{tabular}{|c|c|c|c|c|}
\hline Description & States & $\begin{array}{l}\text { Gain }(\mathrm{dB}) \\
\text { Versus } \\
\text { Uncoded } \\
\text { 8AMPM }\end{array}$ & $\begin{array}{c}\text { Gain }(\mathrm{dB}) \\
\text { Versus } \\
\text { MTCM } \\
\text { 2FSK/8PSK }\end{array}$ & $\begin{array}{c}\text { Gain }(\mathrm{dB}) \\
\text { Versus } \\
\text { TCM } \\
\text { 16QAM }\end{array}$ \\
\hline Rate: ${ }_{6}^{5}$ & 2 & 6.99 & 1.71 & 5.24 \\
\hline \multirow[t]{3}{*}{ Signal Set: CPQFPM $(N=2)$} & 4 & 6.99 & 1.71 & 3.95 \\
\hline & 8 & 6.99 & 0.57 & 3.01 \\
\hline & 16 & 8.75 & 2.32 & 4.01 \\
\hline
\end{tabular}

Alternately, the constant envelope 16-ary CPFPM $(N$ $=4$ ) signal set can be used. This scheme results in a lower throughput due to the larger bandwidth required as compared to the CPQFPM $(N=2)$ scheme. As long as the interset distances do not come into play, the two signal sets have the same values of $d^{2}$ (free), $L_{\min }$, and $P$. With an increasing number of states set partitioning follows a tree structure, as described for some of the AWGN channel codes. For the FPM signals, this procedure does not reduce $^{3}$ the branch distance product $P$.

As shown in Table VI, this code demonstrates improved performance over the rate $\frac{5}{6}$ code using $2 \mathrm{FSK} /$

${ }^{3}$ For 16PSK signals, set partitioning for an increasing number of states using a tree structure yields lower values of branch distance products.
8PSK signals [5], yielding coding gains in $d^{2}$ (free) of $1.71 \mathrm{~dB}$ for the two-state and four-state trellises, and 0.57 $\mathrm{dB}$ for the eight-state trellis. Coding gains in $P$ up to 3.01 $\mathrm{dB}$ for the eight-state trellis are observed. Coding gains up to $8.75 \mathrm{~dB}$ are observed with respect to uncoded 8AMPM ( $r=3 \mathrm{~b} / \mathrm{s} / \mathrm{Hz})$ and up to $5.24 \mathrm{~dB}$ versus rate $\frac{3}{4} \mathrm{TCM} / 16 \mathrm{QAM}(r=3 \mathrm{~b} / \mathrm{s} / \mathrm{Hz})$.

3) Rate $\frac{6}{7}$ code $(k=2)$

AWGN channel design: A CPQFPM $(N=2)$ signal set is used. Here, another method of set partitioning often used for multidimensional signals [19] is illustrated. After the first level of partitioning into two subsets of evennumbered (subset $\mathrm{A}$ ) and odd-numbered (subset $\mathrm{B}$ ) signals, the Cartesian products $\left(A_{0}=A \times A, B_{0}=B \times B\right)$ of elements within these subsets yield 128 unique signal 
TABLE VII

Coding Gains in $d^{2}$ ( free ) FOR The TRELlis-Coded RATE $\frac{6}{7}(k=2)$ QFPM SChemE

\begin{tabular}{lccc}
\hline \multicolumn{1}{c}{ Description } & States & $\begin{array}{c}\text { Gain }(\mathrm{dB}) \\
\text { Versus } \\
\text { Uncoded 8AMPM }\end{array}$ & $\begin{array}{c}\text { Gain (dB) } \\
\text { Versus } \\
\text { TCM/16QAM }\end{array}$ \\
\hline Rate: $\frac{6}{7}$ & 2 & 3.98 & 2.3 \\
Signal Set: CPQFPM $(N=2)$ & 4 & 6.99 & 3.95 \\
& 8 & 6.99 & 3.01 \\
& 16 & 6.99 & 2.25 \\
\hline
\end{tabular}

pairs. Alternately, sets $A$ and $B$ may be comprised of subsets $A A_{0^{-}}-A A_{7}$ and $B B_{0^{-}}-B B_{7}$, respectively, each with eight elements, which are generated as follows:

$$
A A_{0}= \begin{cases}0 & 0 \\ 2 & 2 \\ 4 & 4 \\ 6 & 6 \\ 8 & 8\end{cases}
$$

$$
\begin{aligned}
& A A_{1}=A A_{0}+\left[\begin{array}{ll}
0 & 2
\end{array}\right] ; \quad A_{2}=A A_{0}+\left[\begin{array}{ll}
0 & 4
\end{array}\right] ; \\
& A A_{3}=A A_{0}+\left[\begin{array}{ll}
0 & 6
\end{array}\right] ; \quad A A_{4}=A A_{0}+\left[\begin{array}{ll}
0 & 8
\end{array}\right] ; \\
& A A_{5}=A A_{0}+\left[\begin{array}{ll}
0 & 10
\end{array}\right] ; A A_{6}=A A_{0}+\left[\begin{array}{ll}
0 & 2
\end{array}\right] ; \\
& A A_{7}=A A_{0}+\left[\begin{array}{ll}
0 & 14
\end{array}\right] ; B B_{0}=A A_{0}+\left[\begin{array}{ll}
1 & 1
\end{array}\right] ; \\
& B B_{1}=B B_{0}+\left[\begin{array}{ll}
0 & 2
\end{array}\right] ; \quad B B_{2}=B B_{0}+\left[\begin{array}{ll}
0 & 4
\end{array}\right] ; \\
& B B_{3}=B B_{0}+\left[\begin{array}{ll}
0 & 6
\end{array}\right] ; \quad B B_{4}=B B_{0}+\left[\begin{array}{ll}
0 & 8
\end{array}\right] ; \\
& B B_{5}=B B_{0}+[010] ; B B_{6}=B B_{0}+\left[\begin{array}{ll}
0 & 12
\end{array}\right] ; \\
& B B_{7}=B B_{0}+\left[\begin{array}{ll}
0 & 14
\end{array}\right] \text {. }
\end{aligned}
$$

Clearly, the intraset distance within sets $A_{0}$ and $B_{0}$ is 2, and the interset distance between sets $A_{0}$ and $B_{0}$ is also 2 .

For the two-state trellis, elements from sets $A A_{0}, A A_{2}$, $A A_{4}$, and $A A_{6}$ are assigned to the thirty-two parallel transitions from state 0 to state 0 . Similarly, elements from sets $A A_{1}, A A_{3}, A A_{5}$, and $A A_{7}$ are assigned to the thirtytwo parallel transitions from state 0 to state 1 ; elements from sets $B B_{0}, B B_{2}, B B_{4}$, and $B B_{6}$ are assigned to the transitions from state 1 to state 0 ; and elements from sets $B B_{1}$, $B B_{3}, B B_{5}$, and $B B_{7}$ are assigned to the transitions from state 1 to state 1 . The distance between the parallel transitions determines $d^{2}$ ( free) $(=2)$ for the code.

For the four-state code, the minimum distance between the sixteen parallel transitions accounts for $d^{2}$ ( free $)=4$. For the eight-state trellis, the sixteen sets $A A_{0^{-}}-A A_{7}$ and $B B_{0^{-}}-B B_{7}$ are assigned to transitions emanating from alternate states. The intraset distance of these subsets is equal to 4 , and again $d^{2}$ ( free) is limited by the parallel transitions. The two-branch error event also has the same squared Euclidean distance.

The sixteen-state trellis with four parallel transitions continues to maintain the same distance between parallel transitions. Higher values of $d^{2}$ (free) can be expected when 256 or more states are reached, i.e., when the twobranch error event with the same value as $d^{2}$ ( free) ceases to exist.

Again, for this scheme, $L_{\min }$ and $P$ are determined by the parallel transitions.

This code may be compared with the rate $\frac{3}{4}$ code presented here. The four-state trellis in this code has a smaller $d^{2}$ ( free) than the rate $\frac{3}{4}$ code, due to the distance between the parallel transitions remaining at 2 rather than increasing to 4 , as for the rate $\frac{3}{4}$ code. It is also compared with trellis-coded 2FSK/8PSK and trellis-coded 16QAM in Table VII.

4) Rate $\frac{7}{8}$ code $(k=2)$

AWGN channel design: Here the CPQFPM $(N=2)$ signal set is used. For the rate $\frac{6}{7}$ code requiring 128 signal pairs, the Cartesian cross-products of sets $A$ and $B$ were not required. This code requires 256 unique signal pairs, which are generated by Cartesian products of sets $A$ and $B$ with themselves as illustrated for the rate ${ }^{6}$ code, and by Cartesian cross-products of sets $A$ and $B$, which results in sets $A B_{0^{-}}-A B_{7}$ and $B A_{0^{-}}-B A_{7}$.

For the two-state trellis, there are 64 parallel transitions. In the assignment shown in Fig. 3, set $A$ is comprised of sets $A A_{0}$ to $A A_{7}$, and set $B$ is comprised of sets $B B_{0}$ to $B B_{7}$ given for the rate $\frac{6}{7}$ code. Set $C$ is comprised of sets $A B_{0}$ to $A B_{7}$, and set $D$ is comprised of sets $B A_{0}$ to $B A_{7}$. The distance between the parallel transitions determines $d^{2}($ free $)=2$. The two-branch error event path has $d^{2}=3$. For the four-state trellis, $d^{2}$ (free) is again 2 , determined by the distance between parallel transitions. For the eight-state trellis with 16 parallel transitions and for the sixteen-state trellis with 8 parallel transitions, $d^{2}$ (free) is that due to the two-branch error event.

The CPFPM $(N=4)$ signal set can be used in place of the 16-ary CPQFPM $(N=2)$ signal set. As long as the parallel transitions determine $d^{2}$ (free), the values of $d^{2}$ (free) are the same as that for the rate $\frac{6}{7}$ and $\frac{7}{8}$ CPQFPM $(N=2)$ codes. Only when the intersubset distances begin to play a role with error events of three branches or more (the two-branch error event will also yield the same values of $d^{2}$ (free) as a CPQFPM $(N=2)$ signal set), the 
CPFPM $(N=4)$ signal set offers higher gains than the CPQFPM $(N=2)$ signal set.

Fading channel design: With 64 parallel transitions in the two-state trellis, this code requires a CPQFPM $(N$ $=4)$ signal set. A Cartesian product of this 64 -ary signal set yields 2048 signal pairs, of which only 256 are required. With set partitioning carried out to meet the fading channel criteria of $L_{\min }=k$, the $d^{2}$ (free) obtained for this code remains the same as that obtained for the rate $\frac{5}{6}$ code for the fading channel. Only smaller values of $P$ are encountered. As explained for the AWGN channel design of this code, the values of $d^{2}$ ( free) and $P$ increase when the number of states is made very large.

\section{Discussion}

In this paper, conventional (TCM) and multiple (MTCM) trellis-coding techniques for the AWGN channel and the Rician fading channel are applied to quadrature frequency/phase modulated signals. A $2 N$-dimensional signal space is generated by the quadrature (cosine and sine) components at $N$ orthogonal frequencies. This signal space is used to generate $2 N$-ary $N F S K / 4 P S K$ CEQFPM signal sets and $2 N^{2}$-ary QFPM signal sets (1). The latter signal sets make a more efficient use of the signal space by increasing the number of signals by a factor of $N$. The signal space for $N=2$ is defined in (3).

As shown in Tables II-VII, coding gains are observed when compared to a variety of uncoded and coded modulation schemes. The AWGN channel design for MTCM schemes has a lower value of $L_{\min }$ when compared to the fading channel design. It is observed that, in Table III, the fading design of MTCM schemes is optimum for the AWGN channel as well. This is consistent with the observation in [5] that this is indeed the case with FPM signal sets. As discussed in [5], the simultaneous optimization is advantageous for the use of trellis codes on the Rician fading channel. Coding gains in $d^{2}$ (free) and $P$ are observed in comparison to MTCM schemes reported in literature.

It was observed that the TCM and MTCM schemes with QFPM signals provided coding gains over equivalent schemes employing $\mathrm{FPM}^{4}$ signal sets and MPSK signal sets, as illustrated by a few examples below. Trellis-coded FPM schemes [4], [5] with $N=2$ were observed to provide coding gains over trellis-coded MPSK schemes. The rate $\frac{2}{3} \mathrm{TCM} /[\mathrm{CEQFPM}(N=2)]$ scheme and the rate $\frac{4}{5}$ MTCM/[CEQFPM $(N=2)]$ scheme presented in Section III were first discussed in [4] and [5], respectively, and are given here only for completeness. For the rate $\frac{3}{4}$ TCM scheme in [4] and the rate $\frac{5}{6}$ MTCM scheme in [5], a 2FSK/8PSK signal set was used. The value of $d_{\min }^{2}(=1)$ for the QFPM $(N=2)$ signal set employed for the schemes in Section III is larger when compared to a 2FSK/ 8PSK signal set $\left(d_{\min }^{2}=0.5858\right)$. This is reflected in the

${ }^{4}$ Note that not all FPM signal sets can be classified as QBOM signals. Only NFSK/4PSK FPM signals belong to the class of QBOM signals. improved performance of trellis-coded 2QFPM signals when compared to trellis-coded 2FSK/8PSK signals (and 16PSK signals), demonstrated by coding gains for the rate $\frac{3}{4}$ TCM scheme and the rate $\frac{5}{6}(k=2)$ MTCM scheme, in Tables IV and VI, respectively. Coding gains are also given in Table IV for the rate $\frac{3}{4}$ code versus uncoded 8PSK and TCM/16QASK.

For the two-state trellis, the rate $\frac{5}{6} \mathrm{QFPM}(N=2)$ code has a coding gain of $2.3 \mathrm{~dB}$ over the rate $\frac{5}{6} 2 \mathrm{FSK} / 8 \mathrm{PSK}$ code [5], with similar gains as the number of states increases. With respect to the rate $\frac{5}{6}$ MTCM scheme (AWGN design) using 16QASK signal sets [2], the coding gain for the two-state trellis is $4.32 \mathrm{~dB}$. The value of branch distance product $P$ is also higher with a gain of $1.06 \mathrm{~dB}$ w.r.t. the 2FSK/8PSK code.

The fading design has not been discussed for the rate $\frac{6}{7}$ MTCM scheme, for lack of a 32-ary QFPM signal set to suit the requirement of $L_{\min }=k$ for the 32 parallel transitions in the two-state trellis of this code. If 32 signals from the 64-ary QFPM $(N=2)$ signal set are employed, the values of $d^{2}$ (free) and $P$ will be the same as reported for the rate $\frac{7}{8}$ MTCM scheme, with a lower bandwidth efficiency.

Comparing the TCM and MTCM schemes of equal throughput rates, it is observed that the rate $\frac{2}{3}$ TCM scheme and the rate $\frac{4}{5}$ MTCM scheme utilize the same CPFPM $(N$ $=2$ ) signal sets. The fading design of the latter scheme provides a larger value of $d^{2}$ ( free) for the sixteen-state trellis. Note that for the eight- and sixteen-state trellises, the value of $L_{\min }$ is larger for the TCM scheme, providing better performance on the Rayleigh fading channel. When TCM trellises have no parallel transitions and MTCM trellises have them for a given number of states, the TCM scheme may have a larger value of $L_{\min }$. This result also applies to TCM and MTCM schemes employing MPSK signals. Also, the rate $\frac{5}{6}$ TCM scheme employs QFPM ( $N$ $=4$ ) signals, while the rate $\frac{5}{6}$ MTCM (fading) scheme employs QFPM $(N=2)$ signals. The latter scheme performs better than the former in $d^{2}$ (free), $L_{\min }$, and $P$. The rate $\frac{3}{4}$ TCM scheme and the rate $\frac{6}{7}$ MTCM (AWGN) scheme employ QFPM $(N=2)$ signal sets, and the latter scheme has the same or improved performance compared to the former.

Most importantly, it has also been shown that the continuous phase QFPM (CPQFPM) scheme [14] may provide vastly improved bandwidth efficiency over QFPM. For the $99 \%$ bandwidth criterion, the bandwidth efficiency of uncoded CPQFPM $(N=2)$ is $0.97 \mathrm{~b} / \mathrm{s} / \mathrm{Hz}$ while that of 16-ary PSK is $0.2 \mathrm{~b} / \mathrm{s} / \mathrm{Hz}$. This is also true of the continuous phase FPM (CPFPM) schemes [17]. The use of the continuous phase versions of QFPM and CE-QFPM schemes will affect the bandwidth efficiency without altering the coding gains of the TCM and MTCM schemes discussed in this paper.

While the QFPM signal set is a constant energy signal set, its envelope is not constant over a symbol interval. In this sense, it is different from the nonconstant envelope QAM signal set that it has been compared with, since the 
QAM signal sets do not contain constant energy signals. From this point of view, the QFPM signal sets are expected to be more robust to satellite transponder nonlinearities when compared to QAM signal sets. The CPFPM scheme, which offers coding gains along with its properties of constant envelope, constant energy, continuous phase, and good bandwidth efficiency offers a very good modulation choice.

\section{REFERENCES}

[1] E. Biglieri, D. Divsalar, P. J. McLane, and M. K. Simon, Introduction to Trellis Coded Modulation with Applications. New York: Macmillan, 1991

[2] G. Ungerboeck, "Channel coding with multilevel/phase signals," IEEE Trans. Inform. Theory, vol. 28, pp. 55-67, Jan. 1982

[3] M. K. Simon and D. Divsalar, "The performance of trellis coded multilevel DPSK on a fading mobile satellite channel, "IEEE Trans. Vehic. Technol., vol. 37, May 1988.

[4] R. Padovani and J. K. Wolf, "Coded phase/frequency modulation," IEEE Trans. Commun., vol. COM-34, pp. 446-453, May 1986.

[5] S. S. Periyalwar and S. Fleisher, "Multiple trellis coded frequency and phase modulation," IEEE Trans. Commun., pp. 1038-1046. June 1992.

[6] G. Ungerboeck, "Trellis-coded modulation with redundant signal sets part II: State of the art," IEEE Commun. Mag., vol. 25, pp. 12-21, Feb. 1987.

[7] G. D. Forney, R. G. Gallager, G. R. Lang, F. M. Longstaff, and S. U. Qureshi, "Efficient modulation for band-limited channels," IEEE J. Select. Areas Commun., vol. SAC-2, pp. 632-647, Sept. 1984.

[8] L. F. Wei, "Trellis-coded modulation with multidimensional constellations," IEEE Trans. Inform. Theory, vol. IT-33, pp. 483-501, July 1987.

[9] D. Divsalar and M. K. Simon, "Multiple trellis coded modulation," IEEE Trans. Commun., vol. COM-36, pp. 410-419, Apr. 1988.

[10] —, "The design of trellis coded MPSK for fading channels: Performance criteria," IEEE Trans. Commun, vol. 36, pp. 1004-1012, Sept. 1988.
[11] —, "The design of trellis coded MPSK for fading channels: Set partitioning for optimum code design," IEEE Trans. Commun., vol. 36, pp. 1013-1021, Sept. 188.

[12] S. S. Pietrobon, R. H. Deng, A. LaFanechere, G. Ungerboeck, and D. J. Costello, "Trellis-coded multidimensional phase modulation," IEEE Trans. Inform. Theory, vol. 36, pp. 63-88, Jan. 1990

[13] S. Benedetto, E. Biglieri, and V. Castellani, Digital Transmission Theory. Englewood Cliffs, NJ: Prentice-Hall, 1987.

[14] S. Qu, "New modulation schemes: Principles and performance analysis," Ph.D. Thesis, 1991

[15] S. Fleisher and S. Qu, "Quadrature bi-orthogonal modulation," Patent Application No. 2041279, Canada, Apr. 1991.

[16] S. Fleisher, S. Qu, and S. S. Periyalwar, "Quadrature biorthogonal modulation," in Proc. Canad. Conf. Elect. Comput. Eng., 1991

[17] S. Fleisher and S. Qu, "Multifrequency minimum shift keying," IEEE J. Select. Areas Commun., this issue. pp. 1242-1252.

[18] W. C. Lindsey and M. K. Simon, Telecommunication Systems Engineering. Englewood Cliffs, NJ: Prentice-Hall, 1973.

[19] D. Divsalar and M. K. Simon, "Generalized multiple trellis coded modulation (MTCM)," in IEEE Glob. Telecommun. Conf. Rec., Tokyo, Japan, Nov. 1987.

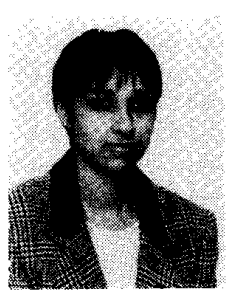

Shalini S. Periyalwar ( $S^{\prime} 88-M^{\prime} 91$ ) received the $B$. Eng. degree in electrical engineering from Bangalore University, Bangalore, India, the M.A.Sc degree in biomedical signal processing and the Ph.D. degree in digital communications from the Technical University of Nova Scotia, Halifax, Canada, in 1987 and 1991.

She is currently an Assistant Professor in the Burchill Communications Research Group at the Technical University of Nova Scotia. Her current research interest is in the area of digital commu-

nications.

Solomon Fleisher ( $M^{\prime} 73-S^{\prime} 80$ ), for photograph and biography, see this issue, p. 1252. 